\title{
The Impact of COVID-19 on Plastic Surgery Residency Training
}

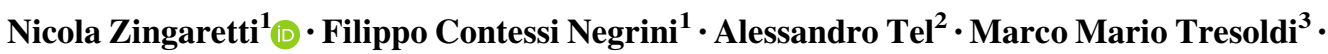 \\ Vittorio Bresadola $^{4}$ Pier Camillo Parodi ${ }^{1}$
}

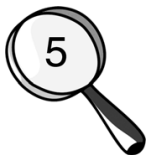

Received: 11 May 2020/Accepted: 11 May 2020/Published online: 26 May 2020

(C) Springer Science+Business Media, LLC, part of Springer Nature and International Society of Aesthetic Plastic Surgery 2020

\begin{abstract}
Nowadays didactic and surgical activities for residents in the surgery field are less and less due to an increasing burden of documentation and "non-educational work." Considering the current lockdown due to the COVID-19 pandemic, it has never been so important to find different ways to allow residents to improve their knowledge. We asked all plastic and esthetic surgery residents in our country to fill out a questionnaire to investigate changes in their didactical activity and analyze problems about their professional growth in the last few months. From the results of such questionnaires, we found that most of the residents feel the decrease in surgical activities during this time is a detrimental factor for their training and that even if all the schools have changed their didactical activities no school has introduced the use of virtual simulators to compensate for the decrease in surgical practice. Actually, the majority of residents use webinars to keep updated, stating that such technologies are useful but not sufficient to analyze plastic surgery topics in depth during COVID-19 lockdown. Virtual interactive tools are well known in different clinical and surgical specialties, and they are considered as a valid
\end{abstract}

Nicola Zingaretti

zingarettin@gmail.com

1 Clinic of Plastic and Reconstructive Surgery, Department of Medical Area (DAME), Academic Hospital of Udine,

University of Udine, Udine, Italy

2 MaxilloFacial Surgery Department, Academic Hospital of Udine, University of Udine, Udine, Italy

3 Department of Clinical Surgical, Diagnostic and Pediatric Sciences, University of Pavia, Pavia, Italy

4 General Surgery Department, Academic Hospital of Udine, University of Udine, Udine, Italy support, but it seems that in plastic surgery they are not so used. According to the most recent studies about residents' didactical program, we have investigated the potential of Anatomage Table in combination with Touch Surgery application as physical and mental aids to bypass the decreased number and kind of surgical interventions performed in this particular time. Anatomage is an academic user-friendly touch screen table; it is used by both medical students and residents to learn human anatomy and to master surgical anatomy. Touch Surgery is an application available on smartphones and tablets that gives the possibility to watch real and virtually designed surgical videos, accompanied by explanatory comments on the surgical phases; they are interactive and give the possibility to check what you have learned through tests administered after virtual classes. In our opinion, these tools represent reliable solutions to improve plastic residents' training, mostly during the COVID-19 pandemic.

Level of Evidence $V$ This journal requires that authors assign a level of evidence to each article. For a full description of these Evidence-Based Medicine ratings, please refer to the Table of Contents or the online Instructions to Authors www.springer.com/00266.

Keywords Questionnaire - Training program · Residents · Education · COVID-19

\section{Dear Sir,}

Nowadays didactive and surgical activities for residents in the surgical field are less and less due to the increasing burden of documentation and "to spend time at non-educational work" that detracts from clinical experience [1]. Most of all, in this difficult period of COVID-19 infection, 
in which the majority of plastic surgery operations are stopped (except for reconstruction procedures after tumor excision or trauma), analogue limitations are applied to classes, congresses and courses; hence, for residents there are less possibilities to improve any skills in their work. In fact, different schools of surgical specialties have been forced to rearrange their daily working activities, reducing the quantity and typology of surgery in order to face the COVID-19 emergence. During this reduction, there has been a decrease in didactical and professional growth of their residents.

All Italian plastic surgery residents were asked to fill out a questionnaire to investigate how their practical and theatrical activity has changed (Table 1).

One hundred and fifteen residents ( $72 \%$ of all plastic residents of our country) answered this questionnaire (Fig. 1). From the answers, it was found that most of the residents feel the lack of training during this time is a detrimental factor for their professional growth. Results of questionnaires show that most Italian schools had to change their didactical program approach following the restrictions due to the pandemic.

Sixty-seven residents claimed that the amount of didactic education has increased with near daily virtual grand rounds and educational conferences.

Fifty-two percent of interviewed residents considered useful but not sufficient the didactical tools available to study and deepen topics concerning plastic surgery available in COVID-19 time. The majority of them (58\%) have heard about virtual interactive tools, but they never used them.

Given that, it is necessary to find smart alternatives to optimize time dedicated to real formative activities in order to keep on maintaining the good quality of teaching and learning target.

There is emerging evidence suggesting that virtual interactive tools may be useful and accessible to support early academic development [2, 3]. In fact, different studies have been published about resident learning principles, stating that the more the process is both physical and mental, the more it is efficient $[2,3]$.

Virtual tools are already widely used by many other specialties such as neurosurgery or maxillofacial surgery, but they are not very common in plastic surgery yet.

The Anatomage Table (San Jose, CA, USA) is a handson three-dimensional educational and diagnostic tool that enables residents to virtually dissect a digital life-sized human cadaver [4]. It is an academical user-friendly instrument by which students and residents can learn anatomy, medical and surgical science's fundamental pillars, in addition to the classical didactic tools, and it is expected to be a valid alternative especially in countries where cadaver dissections are not allowed.
Thanks to the Anatomage Table, plastic surgery residents can build an improved visual perception of the human body by studying the topographic anatomy on virtual rendering and getting more important knowledge of the layered anatomy using the different filters available on the software. It is an excellent support to learn threedimensional realistic anatomy allowing to explore in a very high resolution even those districts that are usually not handled in everyday work; it provides different filters you can use to focus on particular structures such as vascular anatomy. Additionally, the use of this tool does not involve ethical issues.

Although there are other software systems that allow to learn anatomy in a virtual way (i.e., Netter Anatomy flash cards, Skin Atlas, Grey's Anatomy, etc.), Anatomage Table provides the possibility to dissect a $3 D$ model with high-resolution rendering for the cadaveric body.

This table permits to perform virtual cuts of the cadaveric anatomy in order to visualize all the desired cross sections of any anatomical region. Additionally, its high interoperability with the In Vivo software allows to create personalized plans in which STL (Standard Tessellation Language) files can be imported and overlapped on the highly accurate anatomical renderings. Anatomage Table works as an advanced DICOM viewer, as the computational algorithms allow for a realistic three-dimensional representation of CT and MRI scans, with remarkable capabilities to reconstruct complex structures such as arteries and veins.

The second important virtual tool for plastic surgery residents is Touch Surgery. It is an online platform available for both iOS and Android devices which offers a wide range of surgical interventions in different specialties [5]. There are forty-two procedures available for plastic surgery. This app aims to improve surgical and procedural readiness among healthcare providers; furthermore, it is proving to be a valid aim to help residents to keep on learning surgical interventions; moreover, it helps to visualize in a step-by-step fashion every single movement in surgical procedures.

Our experience allowed us to notice some strengths and weaknesses in both didactical systems (Table 2(a), (b)).

In our opinion, virtual tools are not going to replace classical didactical items, but they can integrate them to help the students and residents to be more confident, stimulating positive emotional and motivational responses and simultaneously allowing to test their own preparation and growth.

These systems represent a suitable way to maintain training among plastic surgery residents; more research is needed to evaluate both the potential of educational apps to support early learning and their limitations. 
Table 1 Questionnaire for plastic surgery residents during the current lockdown due to COVID-19 pandemic

1. As a plastic resident, how do you think the total training time should be divided between practice and theory?
(a) $25 \%$ practice, $75 \%$ theory
(b) $50 \%$ practice, $50 \%$ theory
(c) $75 \%$ practice, $25 \%$ theory
(d) Other

2. During COVID-19, how much did elective surgery activity decrease in your department?
(a) Decrease of $0-25 \%$
(b) Decrease of $25-50 \%$
(c) Decrease of $50-75 \%$
(d) Decrease of $75-100 \%$

3. Do you think the decrease in training activity (both didactical and practical) will affect your training and professional growth?
(a) $\mathrm{No}$
(b) A little
(c) Yes
(d) A lot

4. How has the didactical program offered by your school changed during COVID-19?
(a) We have no didactical programs
(b) Didactical activity is reduced compared to pre-COVID
(c) Didactical activity is the same compared to pre-COVID
(d) Didactical activity is increased compared to pre-COVID

5. Which studying methods do you usually use?
(a) Books and scientific papers
(b) Webinars, classes and courses organized by my school or other societies
(c) Virtual didactical tools (i.e., anatomical table, didactical softwares)
(d) Others

6. Which studying methods do you use during COVID-19?
(a) Books and scientific papers
(b) Webinars, classes and courses organized by my school or other societies
(c) Virtual didactical tools (anatomical table, didactical softwares)
(d) Others

7. Currently, advanced didactical tools in use (e.g., surgery didactical software, virtual anatomical tables, etc.) allow residents and senior doctors to deeper investigate topics of interest (e.g., anatomy, planning of surgery, etc.). Have you ever tried these tools?
(a) No, I don't know them
(b) I heard about them, but never used them
(c) I rarely use them
(d) Yes, I usually use them

8. How do you evaluate the didactical tools you can use during COVID-19? Are they adequate to train on plastic operations?
(a) Not adequate
(b) Useful, but not sufficient
(c) Sufficient
(d) Excellent

9. Are the didactical tools you usually use adequate to train and prepare the operations you can't see and perform in your school? Do you feel prepared enough to do them?
(a) Not at all
(b) Not much
(c) Yes, I am able enough
(d) Yes, I am totally prepared 


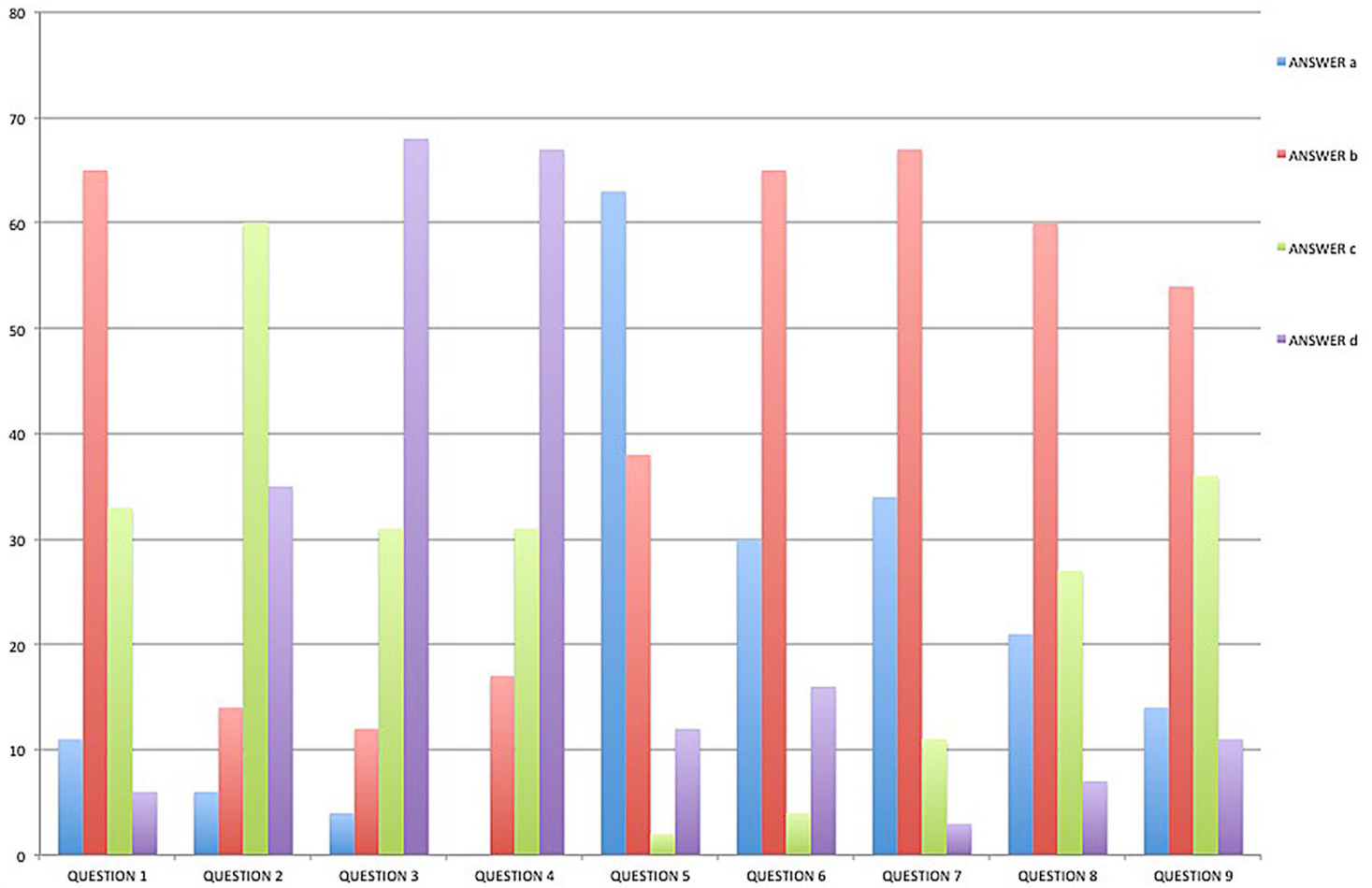

Fig. 1 Outcomes of questionnaire survey

Table 2 Positive and negative sides of programs. (a) The Anatomage Table gives students the ability to virtually dissect a digital human cadaver, navigate 3D cross-sectional anatomy of a virtual cadaver and acquire images needed for projects or presentations. It is expensive. (b) Touch Surgery provides a hands-on experience for learners to improve knowledge of the steps of an operation; it is a user-friendly platform. Few amounts of available procedures
Strengths
Weaknesses

\section{(a) Anatomage Table}

Realistic anatomy on demand

Freedom of action

Realistic 3D rendering in scale 1:1

Different filters with which explore different structures without interferences

Availability of radiologic and pathologic anatomy

Patient-safe learning tool

Test mode available

No ethical issues

(b) Touch Surgery

Low cost (right now for free due to COVID-19)

User-friendly

\section{Didactical}

Comfortable and usable anywhere

Test mode available

Wide variety of surgical specialties to practice

Patient-safe learning tool

No ethical issues
It costs approximately $\$ 80000$

No children's anatomy

It is heavy and difficult to move

Access restricted to one person per time

It loose some small details, such as in peripheral nerves

Need to be update

Limited number of surgical intervention (42 for plastic surgery) Few esthetic intervention

No freedom of action

Some videos are virtual-made, less realistic

Need update in case of new surgical techniques 


\section{Compliance with Ethical Standards}

Conflict of interest The authors declare that they have no conflicts of interest to disclose.

Statement of Human and Animal Rights This article does not contain any studies with human participants or animals performed by any of the authors.

Informed Consent For this type of study, informed consent is not required.

\section{References}

1. Papas A, Montemurro P, Heden P (2018) Aesthetic training for plastic surgeons: are residents getting enough? Aesth Plast Surg 42:327-330
2. Ekstrand C, Jamal A, Nhuyen R, Kudryk A, Mann J, Mendez I (2018) Immersive and interactive virtual reality to improve learning and retention of neuroanatomy in medical students: a randomized controlled study. CMAJ Open 6:E103-E109

3. Griffith SF, Hagan MB, Heymann P, Heflin BH, Bagner DM (2020) Apps as learning tools: a systematic review. Pediatrics 145:e20191579

4. Anatomage table. Anatomage website: http://www.anatomage. com/anatomage-medical/. Published 2016. Accessed 23 Oct 2017

5. Bunogerane GJ, Taylor K, Lin Y, Costas-Chavarri A (2018) Using touch surgery to improve surgical education in low- and middleincome settings: a randomized control trial. J Surg Educ. $75: 231-237$

Publisher's Note Springer Nature remains neutral with regard to jurisdictional claims in published maps and institutional affiliations. 